\title{
Dual-Level Game-Based Energy Efficiency and Fairness for Green Cellular Networks
}

\author{
Sungwook Kim \\ Department of Computer Science, Sogang University, 35 Baekbeom-ro (Sinsu-dong), Mapo-gu, Seoul 121-742, Republic of Korea
}

Correspondence should be addressed to Sungwook Kim; swkim01@sogang.ac.kr

Received 28 January 2016; Revised 25 April 2016; Accepted 4 May 2016

Academic Editor: Laurence T. Yang

Copyright (C) 2016 Sungwook Kim. This is an open access article distributed under the Creative Commons Attribution License, which permits unrestricted use, distribution, and reproduction in any medium, provided the original work is properly cited.

In the recent decades, cellular networks have revolutionized the way of next generation communication networks. However, due to the global climate change, reducing the energy consumption of cellular infrastructures is an important and urgent problem. In this study, we propose a novel two-level cooperative game framework for improving the energy efficiency and fairness in cellular networks. For the energy efficiency, base stations (BSs) constantly monitor the current traffic load and cooperate with each other to maximize the energy saving. For the energy fairness, renewable energy can be shared dynamically while ensuring the fairness among BSs. To achieve an excellent cellular network performance, the concepts of the Raiffa Bargaining Solution and Jain's fairness are extended and practically applied to our dual-level cooperative game model. Through system level simulations, the proposed scheme is evaluated and compared with other existing schemes. The simulation results show that our two-level game approach outperforms the existing schemes in providing a better fair-efficient system performance.

\section{Introduction}

The current explosive popularity of smartphones and mobile devices has ignited surging traffic demands for wireless accesses and has been incurring massive energy consumption, which causes global warming due to $\mathrm{CO}_{2}$ emissions. With the increasing awareness of global warming and environmental consequences of Information and Communications Technology (ICT), researchers have been seeking ways to reduce energy consumption. As a significant component of ICT energy consumption, cellular network system will have greater economic and ecological impact in the coming years. Concentrating on environmental influences, a new research area called "green cellular networks" has recently emerged. This green approach can give extra commercial benefits, mainly by reducing the operating expense related to energy cost [1-3].

In wireless cellular networks, energy consumption is mainly drawn from base stations (BSs); they account for more than 50 percent of the cellular network's energy consumption. In addition, the number of BSs is expected to be doubled by 2012 [4]. Therefore, promising technology has been developed to improve the energy efficiency in BSs; it is crucial to implement green cellular networks while approximating an optimal energy saving. From the perspective of cellular network management, the energy-efficient operation of BSs is not only a matter of social environmental responsibility but also tightly related to cellular network control issues [4-7].

Motivated by the above discussion, in this study, our main focus is devoted to maximizing the energy efficiency for BSs. To address the challenge of increasing energy efficiency and profitability in future green cellular networks, we consider various paradigm-shifting methodologies [1, 4]. Among all the promising energy saving approaches, BS sleeping and renewable energy (RE) distribution methods are very effective and prominent solutions to optimize the energy utilization in green cellular networks [4-6]. The past decade has seen a surge of research activities in each individual method. However, little research has been done for the proper combination of these two methods.

The intuitive idea of BS sleeping method is to switch off the BSs when the traffic load is below a certain threshold during a certain time period. Simply, the BS sleeping problem can be formulated as an optimization problem that minimizes the number of active BSs while supporting the traffic load in a cellular network. This problem is a well-known combinatorial 
optimization problem [4]. However, the BS sleeping problem has been proven to be NP-hard. Moreover, solving this problem generally requires a central controller as well as the global information, which makes the problem more challenging [1].

Another leading approach for green cellular networks is the use of RE [5, 6]. In recent years, RE resources, such as solar panels and wind turbines, can be considered as complementary energy providers for conventional power systems, such as diesel generators or the power grid. Due to the significantly higher cost of conventional power generation, RE becomes more and more attractive. However, although RE sources are attractive for green cellular network operations, they suffer from higher variability as compared to conventional energy sources [5]. Therefore, a key consideration for the $\mathrm{RE}$ management is to maximize the energy efficiency while compensating the variability of the RE source.

Under widely dynamic cellular system conditions, BSs and RE Providers (REPs) can be assumed as intelligent rational decision makers, and they select a best-response strategy to maximize their expected payoffs. This situation is well suited for the game theory. Game theory is a field of applied mathematics that provides an effective tool to model interactions among independent decision makers. It can describe the reactions of one set of decision makers to another and analyze the situations in terms of conflict and cooperation [8]. Therefore, game theory is really useful in analyzing the mutual interactions among BSs and REPs, and it can be a major paradigm to retain an effective solution in green cellular networks.

In order to feature the complex interactive relations of BSs and REPs, we propose a novel energy control scheme for the green cellular network management. To ensure fair-efficient energy provisioning, the methodology adopted in the proposed scheme is a two-level cooperative game model. This approach can improve effectively the energy efficiency and fairness in cellular networks. At the efficient-control stage, some BSs are grouped together as a cluster and work together toward an optimal system performance. When the traffic load of a specific BS is below a certain threshold, this BS is switched off, and running services are taken care of by neighboring BSs that remain active in the cluster. At the fairness-control stage, REPs fairly distribute the available RE to their corresponding BSs. Under dynamically changing network environments, BSs and REPs coordinate with each other in order to ensure the fair-efficient energy management. Therefore, the proposed dual-level game approach is suitable to get a globally desirable network performance while considering the practical implementation in real-world network operations.

This paper is organized as follows. The following section gives an overview on related studies in the literature. Section 3 describes how to implement our two-level game model for green cellular networks. Afterwards, the main steps of the proposed algorithm are presented. In Section 4, performance evaluation results are presented along with comparisons with the existing schemes proposed in $[1,4]$. Through simulation, we show the ability of proposed scheme to achieve high accuracy and promptness in dynamic cellular network environments. Finally, we end up with some concluding remarks in Section 5.

\section{Related Work}

Recently, several BS energy management schemes have been presented for green cellular network systems. The Distributed Energy-Power Control (DEPC) scheme [9] provided a cost and energy saving solution for cellular operators. In the DEPC scheme, two energy-efficient power control algorithms were developed: gradient-based distributed power control algorithm and energy-efficient game-based power control algorithm. The gradient-based approach maximized energy efficiency by macro-BS or femtoaccess points operating in a semiautonomous mode, with partial derivatives and system power information exchanged periodically between neighboring macro-BSs or femtoaccess points. The energy-efficient approach was proposed based on game theory, in which each BS updated its power-allocation strategy to maximize its utility. The existence and uniqueness of equilibrium of the power control game were proved [9].

The Switching and Cell Zooming Design (SCZD) scheme [10] investigated the QoS-aware BS switching and cell zooming, that is, BS power control, problem for green wireless cellular networks. This scheme was developed as a unified cross-layer model that captured interaction between the physical and network layers. By partitioning each cell into cell partitions, the SCZD scheme can explicitly model the intercell interference and location-dependent users' QoSs. This approach designed an efficient BS switching mechanism that can maintain user QoS requirements while exploiting heterogeneous traffic distribution over space and time for energy saving. In addition, the SCZD scheme developed a power control algorithm that can further improve the energy efficiency [10].

The Decentralized Powered Base Station Control (DPBC) scheme [11] considered a green wireless communication system in which base stations were powered by renewable energy sources. This system consisted of a capacity-constrained renewable power supplier and a BS that faced a predictable random connection demand from mobile user equipment. In the DPBC scheme, the BS, which was powered via a combination of a renewable power source and the conventional electric grid, sought to specify the renewable power inventory policy, that is, the power storage level. In addition, $M / M / 1$ make-to-stock queuing model was proposed to investigate the decentralized decisions when the two parties optimized their individual costs in a noncooperative manner. Therefore, the problem was formulated as a noncooperative game whose Nash equilibrium strategies were characterized to identify the causes of inefficiency in the decentralized operation. Moreover, the DPBC scheme provided valuable energy cost savings by allowing the BSs to smartly use a combination of renewable and traditional energy, even when the BS had a heavy traffic of connections [11].

The Distributed Cooperative Base Station Sleeping (DCBS) scheme in [1] was developed as a distributed cooperative scheme for improving the energy efficiency of green cellular networks. To maximize the energy saving, the DCBS scheme tried to select the BS sleeping strategy while guaranteeing users' minimal service requirements. In this approach, the inter-BS cooperation was formulated following the principle 
of ecological self-organization. To capture the network impact of the BS switching operation, an interaction graph was defined, and then the problem of energy saving was formulated as a constrained graphical game, where each BS acted as a game player with the constraint of traffic load [1].

The Hybrid Energy Green Utilization (HEGU) scheme in [4] decomposed the energy saving problem into two subproblems: the multistage energy allocation problem and the multi-BSs energy balancing problem. These two problems were subsequently solved based on the characteristics of the green energy generation and the mobile traffic. Taking advantages of the spatial diversity of the mobile traffic, the HEGU scheme attempted to balance the green energy consumption among BSs so as to reduce the on-grid energy consumption of the cellular network. In the HEGU scheme, individual BSs applied the multistage energy allocation algorithm to optimize the green energy allocation based on the estimation of the mobile traffic and the green energy generation. With the feedback of green energy allocation, the network controller tried to balances the green energy usage among the BSs and determined the BSs cell sizes [4]. All the earlier work has attracted much attention and introduced unique challenges. However, little has been done for the issue of fairness in the green cellular network management.

\section{Proposed Fair-Efficient Energy Control Algorithms}

In this section, we consider a green cellular network powered by the conventional and renewable energy grid. First, we introduce a BS sleeping algorithm for the energy efficiency. And then, RE distribution algorithm is presented for the energy fairness. Finally, we describe the main steps of fairefficient energy control scheme based on the two-level game model.

3.1. Two-Level Game Model for Green Cellular Networks. At present, all BSs in cellular networks are working on the "always-ON" state, regardless of the traffic levels associated with them. Moreover, a traditional on-state BS is designed to satisfy peak traffic requirements. However, in fact, the average peak utilization rates of BSs are merely at $65 \% \sim 70 \%$. This situation motivates many power saving efforts that have been done towards underutilized BSs. Nowadays, BS sleeping strategy is an effective mechanism to reduce energy consumption of cellular networks. Generally, a sleeping BS reduces its energy consumption by 1/2 2/3 compared with its active mode. Therefore, it is reasonable to let a subset of BSs go to sleep when the traffic load is below a certain threshold $[12,13]$.

During cellular system operations, BSs and REPs should make decisions individually. In this situation, a main issue for each agent is how to perform well by considering the mutualinteractive relationship. In this study, the dynamic interactions of BSs and REPs are formulated as a two-level game. At the first level, BSs play an efficient-control game; the BSs dynamically create an ad hoc cluster, and a low traffic load BS is switched off. At this moment, the traffic load in the sleeping
BS is effectively shared through a cooperative manner. At the second level, BSs and REP play a fairness-control game; some BSs are grouped as permanent clusters with one REP, and each REP provides the available RE to its corresponding BSs through a cooperative manner. For the implementation practicality, our proposed scheme is designed in an entirely distributed and self-organizing interactive fashion.

Mathematically, the efficient-control level game $\left(\mathbb{G}^{\mathrm{EC}}\right)$ can be defined as $\mathbb{G}^{\mathrm{EC}}=\left\{\mathbb{N}, \mathscr{C}_{i, i \in \mathbb{N}}, \mathbb{L},\left\{\boldsymbol{S}_{j}\right\}_{j \in \mathbb{N}},\left\{U_{j}\right\}_{j \in \mathbb{N}}, T\right\}$ at each time period $t$ of gameplay.

(i) $\mathbb{N}=\{1, \ldots, n\}$ is the finite set of all BSs; they are game players in $\mathbb{G}^{\mathrm{EC}}$.

(ii) $\mathscr{C}_{i}$ is the ad hoc cluster for the sleeping BS $i$; that is, $i \in \mathbb{N}$. Multiple ad hoc clusters can exist based on the current traffic condition.

(iii) $\mathbb{L}$ is the set of BSs traffic loads in the $\mathscr{C}$. If $m$ BSs exist in $\mathscr{C}, \mathbb{L}$ can be defined as $\mathbb{L}=\left\{l_{1}, l_{2}, \ldots, l_{m}\right\}$.

(iv) $\mathbf{S}_{j}$ is the set of strategies of the BS $j \in \mathscr{C}$. $\mathbf{S}_{j}$ represents the amount of taken traffic load from the sleeping BS in $\mathscr{C}$.

(v) $U_{j}$ is the payoff received by the BS $j$. It is the profit obtained from the BS sleeping algorithm. Usually, it corresponds to the received benefit minus the incurred cost.

(vi) The $T$ is a time period. The $\mathbb{G}^{\mathrm{EC}}$ is repeated $t \in T<$ $\infty$ time periods with imperfect information.

To distribute the RE to BSs, BSs in green cellular networks are also clustered. Compared to the cluster $\mathscr{C}$ in $\mathbb{G}^{\mathrm{EC}}$, these clusters are formed permanently. One REP has its own permanent cluster and each REP is responsible for distributing its RE to the BSs in its corresponding cluster. To formulate interactions between BSs and REPs, the fairness-control game $\left(\mathbb{G}^{\mathrm{FC}}\right)$ can be defined as $\mathbb{G}^{\mathrm{FC}}=\left\{\mathbb{K},\left\{\mathscr{M}^{i}\right\}^{i \in \mathbb{K}},\left\{\mathrm{A}^{i}\right\}^{i \in \mathbb{K}},\left\{\mathscr{U}_{j}^{i}\right\}_{j \in \mathscr{M}^{i}}^{i, \mathbb{K}}\right.$, $T\}$ at each time period $t$ of gameplay.

(i) $\mathbb{K}=\left\{\mathscr{K}_{1}, \ldots, \mathscr{K}_{z}\right\}$ is the finite set of REPs in the green cellular network, where $z$ is the total number of REPs and $\mathscr{K}_{i, 1 \leq i \leq z}$ is the permanent cluster $i$.

(ii) $\mathscr{M}^{i}=\left\{\mathscr{B}_{1}^{i}, \ldots, \mathscr{B}_{w}^{i}\right\}$ is the finite set of BSs in the permanent cluster $i$, where $w$ is the number of BSs in $M^{i}$.

(iii) In our fairness-control game $\left(\mathbb{G}^{\mathrm{FC}}\right), \mathbb{K}$ and $\left\{\mathscr{M}^{i}\right\}^{i \in \mathbb{K}}$ are game players.

(iv) $\mathbb{A}^{i}=\left(\mathscr{A}_{1}^{i}, \mathscr{A}_{2}^{i}, \ldots, \mathscr{A}_{w}^{i}\right)$ is the set of strategies for $\mathscr{M}^{i}$; $\mathrm{A}^{i}$ represents the amount of allocated RE, and it is decided by the REP $\mathscr{K}_{i} \in \mathbb{K}$.

(v) $\mathcal{U}_{j}^{i}$ is the payoff received by BS $j \in \mathscr{M}^{i}$. It is BS $j$ 's profit obtained from the REP $\mathscr{K}_{i}$.

(vi) $T$ is a time period. The $\mathbb{G}^{\mathrm{FC}}$ is repeated $t \in T<\infty$ time periods with imperfect information. 
A simple example of $\mathbb{G}^{\text {EC }}$ can be demonstrated like this; if BS $i$ has a threshold-below traffic load, an ad hoc cluster $(\mathscr{C})$ can be formed. BSs in this cluster take over the traffic load from BS $i$ while getting the payoff based on the cooperative game model. And then, BS $i$ is switched off. When the traffic load increases in BS $i$ area, BS $i$ is switched on. A simple example of $\mathbb{G}^{\mathrm{FC}}$ can be demonstrated like this; if 30 BSs and 5 REPs exist in the cellular system, each REP can cover 6 BSs in a permanent cluster $(\mathscr{M})$. In each cluster, RE is distributed to ensure the fairness among BSs; it is also implemented in a cooperative manner. The notations for our game-based algorithms are given as follows:

$\mathbb{N}$ : the finite set of all BSs,

$\mathscr{C}$ : the ad hoc cluster in the efficient-control level game,

$\mathbb{L}:$ the set of BSs traffic loads in $\mathscr{C}$,

S: the set of strategies of the BS in the efficient-control level game,

$U$ : the payoff received by the BS,

$T$ : a time period for game play,

$\mathbb{K}$ : the finite set of REPs,

$\mathscr{M}$ : the finite set of BSs in the permanent cluster,

A: the set of strategies in the fairness-control level game,

$\mathscr{F}_{j}^{\mathfrak{B}}$ : the fixed power consumption for BS $j$,

$\mathscr{D}_{j}^{\mathfrak{B}}$ : the dynamic power consumption for BS $j$,

$\mathcal{S}^{\mathfrak{B}}$ : BS switching cost including the traffic transferring overhead,

$\widehat{L}_{-i}$ : the transferring traffic load vector from the sleeping BS $i$,

$\Psi_{j}^{\mathfrak{B}}$ : the power consumption in BS $j$,

$\Gamma_{L}, \Gamma_{H}$ : the lower and upper thresholds,

$\mathfrak{P}$ : the incentive-payment vector for BSs,

$\mathscr{F}_{e_{-} v}:$ the economic fairness index,
$\mathscr{F}_{o_{-} l}$ : the overload fairness index,

$M_{-} \mathscr{F}_{\mathscr{M}}^{j}:$ a multiobjective fairness function for $\mathscr{M}^{j}$,

$\beta$ : control parameter to different fairness indexes.

3.2. Efficient-Control Game at the First Stage. In this study, a cellular network deployed in an urban area is considered, and each cell may experience various traffic densities over space and time. Each cell is typically shaped as a hexagonal area, and it is serviced by a BS with a rational operator. For adaptive power control decisions, each BS exchanges local information periodically with the adjacent neighboring BSs. To save the consuming energy, we can switch off the BS with light traffic load while transferring the running services to its neighboring BSs [12]. In general, the power consumption in BS $j\left(\Psi_{j}^{\mathfrak{B}}\right)$ is composed of two types of power consumptions: fixed power consumption $\left(\mathscr{F}_{j}^{\mathfrak{B}}\right)$ and dynamic power consumption $\left(\mathscr{D}_{j}^{\mathfrak{B}}\right)[1,13] . \mathscr{F}_{j}^{\mathfrak{B}}$ denotes the power consumed statically even though a BS is idle. In active BS mode, it includes power cost by power amplifier, feeder, transmit antennas, and air conditioning. $\mathscr{D}_{j}^{\mathfrak{B}}$ mainly denotes the power used for actual data transmission; it is related to the current traffic load in BS $j[1,13]$. In addition, BS switching cost $\left(\mathcal{S}^{\mathfrak{B}}\right)$ occurs for the case of BS sleeping; it is the extra power consumption when the status of BS transforms between active and sleep modes. Depending on the associated traffic load, $\mathcal{S}^{\mathfrak{B}}$ can be estimated by including the traffic transferring overhead.

In the proposed scheme, each BS reports its own traffic load (l) periodically to the Mobile Switching Center (MSC), which works as a network gateway, and is responsible for the intercell management of BSs. When the traffic load of BS $i$ is less than the threshold $(\Gamma)$, BS $i$ and its neighboring BSs dynamically form an ad hoc cluster $\left(\mathscr{C}_{i}\right)$. And then, BS $i$ attempts to transfer its traffic load to the neighboring active BSs in $\mathscr{C}_{i}$. In $\mathscr{C}_{i}$, the transferring traffic load $(\widehat{l})$ from the sleeping BS $i$ is denoted by $\widehat{L}_{-i}=\left[\widehat{l_{1}^{i}}, \widehat{l_{2}^{i}}, \ldots, \widehat{l_{i-1}^{i}}, \widehat{l_{i+1}^{i}}, \ldots, \widehat{l_{m}^{i}}\right]$; $\widehat{L}_{-i}$ is decided geographically. If BS $i$ is switched off, the active BSs in $\mathscr{C}_{i}$ will get paid according to $\widehat{L}_{-i}$. Therefore, the BS sleeping problem can be formulated to an optimization problem as follows; the goal of this problem is to minimize the total power consumption of $\mathscr{C}_{i}$ :

$\min _{\mathbf{s}_{j}} \sum_{j \in \mathscr{C}_{i}} \Psi_{j}^{\mathfrak{B}}$

s.t. $\quad$ if BS $i$ is not switched off, $\Psi_{j}^{\mathfrak{B}}=\mathscr{F}_{j}^{\mathfrak{B}}+\mathscr{D}_{j}^{\mathfrak{B}}$, where all $j \in \mathscr{C}_{i}$

if BS $i$ is switched off, $\begin{cases}\Psi_{j}^{\mathfrak{B}}=\mathscr{F}_{j}^{\mathfrak{B}}+\mathscr{D}_{j}^{\mathfrak{B}}+\mathfrak{T}\left(\widehat{l}_{j}\right), & \text { where } j \in \mathscr{C}_{i}-\{i\} \\ \Psi_{i}^{\mathfrak{B}}=\mathcal{S}_{i}^{\mathfrak{B}}, & \text { where } i \neq j,\end{cases}$

$$
\mathscr{D}_{j}^{\mathfrak{B}}=\eta \times l_{j}, \quad \mathcal{S}_{i}^{\mathfrak{B}}=\varepsilon \times l_{i}, \quad \mathfrak{T}\left(\widehat{l}_{j}\right)=\left(\varepsilon \times \widehat{l}_{j}\right)+\left(\eta \times \widehat{l}_{j}\right),
$$


where $\eta$ and $\varepsilon$ are the energy parameters for the traffic load $(l)$ execution and transferring, respectively. Even though the BS sleeping strategy is essential to the energy efficiency in green cellular networks, frequent BS on/off switching may cause the degradation to the Quality-of-Service (QoS) while increasing the network operational cost. To avoid the frequent mode transitions, we develop a dual-threshold based sleep mechanism. When the traffic load of BS is less than the lower threshold $\left(\Gamma_{L}\right)$, the BS will switch off. When the traffic load of BS reaches the upper threshold $\left(\Gamma_{H}\right)$, the BS will switch on. Our dual-threshold approach can effectively prevent shuttling of BS status between on and off states. Based on the state transition cost and current power consumption, we adaptively adjust two threshold values while minimizing the energy consumption. In this study, $\Gamma_{L}$ and $\Gamma_{H}$ of BS $i$ are defined as follows:

$$
\begin{aligned}
\Gamma_{L}^{i} & =\mathcal{S}_{i}^{\mathfrak{B}}, \\
\Gamma_{H}^{i} & =\frac{1}{\left|\mathscr{C}_{i}\right|} \times \sum_{j \in \mathscr{C}_{i}} l_{j} .
\end{aligned}
$$

If the active BS $i$ 's $\Psi_{i}^{\mathfrak{B}}$ is less than $\Gamma_{L}^{i}$, BS $i$ is switched off. If the traffic load in the sleeping BS $i$ 's area reaches $\Gamma_{H}^{i}$, the BS $i$ will switch on. In this study, the MSC and neighboring BSs are assumed to have the ability of detecting the traffic load in the sleeping BS's area.

To design the BS sleeping algorithm, we should consider how self-interested BSs would agree to serve the transferred traffic load from the sleeping BS. In this study, we use an incentive-payment technique because it can make a self-organizing system effectively functional. For neighboring $\mathrm{BSs}$, the incentive-payment vector $\boldsymbol{B}=$ $\left[\mathfrak{P}_{1}(\cdot), \ldots, \mathfrak{P}_{i-1}(\cdot), \mathfrak{P}_{i+1}(\cdot), \ldots, \mathfrak{P}_{m}(\cdot)\right]$ is provided to induce selfish BSs to participate in the BS sleeping mechanism. To ensure the socially efficient outcome, incentive compatibility, budget balance, and participation constraints, $\boldsymbol{\Re}$ should be dynamically decided. Based on $\boldsymbol{\beta}$, the neighboring BS $j$ 's utility function $\left(U_{j}(\cdot)\right)$ is defined by

$$
\begin{array}{r}
U_{j}\left(\widehat{l_{j}^{i}}, \widehat{\Psi_{j}^{B \mathcal{B}}}\right)=-\left[\left(\Delta_{e} \times\left(\eta \times \widehat{l_{j}^{i}}\right)\right)+\left(\Delta_{t} \times\left(\varepsilon \times \widehat{l_{j}^{i}}\right)\right)\right] \\
+\mathfrak{P}_{j}\left(\widehat{L}_{-i}, \widehat{\Psi_{j}^{\mathfrak{B}}}\right) \\
\text { s.t. } \widehat{L}_{-i}=\left[\widehat{l_{1}^{i}}, \widehat{l_{2}^{i}}, \ldots, \widehat{l_{i-1}^{i}}, \widehat{l_{i+1}^{i}}, \ldots, \widehat{l_{m}^{i}}\right], \\
i \neq j, i, j \in \mathscr{C}_{i},
\end{array}
$$

where $\widehat{l}_{j}^{i}$ is the transferring traffic load from the sleeping BS $i$ to BS $j$ and $\widehat{\Psi_{j}^{\mathfrak{B}}}$ is the extra power consumption for $\widehat{l_{j}^{i}}$. $\Delta_{e}, \Delta_{t}$ are the cost fee parameters for using electricity and transferring overhead for the traffic load $\left(\widehat{l_{j}}\right)$, respectively. $\mathfrak{P}_{j}\left(\widehat{L}_{-i}, \widehat{\Psi_{j}^{\mathfrak{B}}}\right)$ is decided by the MSC based on the traffic distribution information $\widehat{L}_{-i}$. According to the rational participation constraint, $\mathfrak{P}_{j}(\cdot)$ is dynamically decided to guarantee $U_{j}(\cdot) \geq 0$. This condition can translate the selfish motives of BSs into desirable actions for traffic sharing.

In this study, we develop a cooperative game mechanism to decide $\boldsymbol{P}$. The main design goal is to effectively redistribute the saving energy while meeting the rational constraints. To satisfy this goal, our proposed scheme adopts the concept of Raiffa Bargaining Solution (RBS); this solution can ensure the Pareto Optimality, Independence of Linear Transformations, Symmetry, and Monotonicity [12]. To implement the RBS, BS $j$ 's preference function $v_{j}\left(\widehat{L}_{-i}, \widehat{\Psi_{j}^{\mathfrak{B}}}\right)$ is defined with the minimum utility $U_{j}^{\min }\left(\widehat{l_{j}^{i}}, \widehat{\Psi_{j}^{\mathfrak{B}}}\right)$ and maximum utility $U_{j}^{\max }\left(\widehat{l_{j}^{i}}, \widehat{\Psi_{j}^{\mathfrak{B}}}\right)$ :

$$
\begin{aligned}
v_{j}\left(\widehat{L} \widehat{L}_{-i}, \widehat{\Psi_{j}^{\mathfrak{B}}}\right)= & {\left[\left(U_{j}\left(\widehat{l_{j}^{i}}, \widehat{\Psi_{j}^{\mathfrak{B}}}\right)-U_{j}^{\min }\left(\widehat{l_{j}^{i}}, \widehat{\Psi_{j}^{\mathfrak{B}}}\right)\right)+\frac{1}{\left|\mathscr{C}_{i}\right|-2} \times\left(\sum_{k \in \mathscr{C}_{i}, k \neq i, j} U_{k}^{\max }\left(\widehat{l_{k}^{i}}, \widehat{\Psi_{j}^{\mathfrak{B}}}\right)-U_{j}\left(\widehat{l_{j}^{i}}, \widehat{\Psi_{j}^{\mathfrak{B}}}\right)\right)\right]^{\alpha_{j}}, } \\
\text { s.t. } & \sum_{j \in \mathscr{C}_{i}, j \neq i} \alpha_{j}=1,
\end{aligned}
$$

where $U_{j}^{\min }\left(\widehat{l_{j}^{i}}, \widehat{\Psi_{j}^{\text {B }}}\right)$ is expected to be the result if BSs cannot reach an agreement. It is at least guaranteed for the BS $j$ in the cooperative game, that is, zero in our system. $\alpha_{j}$ is the normalized bargaining power. In the proposed scheme, $\alpha_{j}$ is obtained as $\sum_{k \in \mathscr{C}_{i}, k \neq i}\left(\widehat{l}{ }_{j} / \widehat{l}_{k}^{i}\right)$. Given this preference function, we can write the RBS optimization problem as follows:

$$
\begin{aligned}
& \mathfrak{P}^{*}=\left[\mathfrak{P}_{1}^{*}\left(\widehat{L}_{-i}, \widehat{\Psi_{1}^{\mathfrak{B}}}\right), \ldots, \boldsymbol{P}_{i-1}^{*}\left(\widehat{L}_{-i}, \widehat{\Psi_{i-1}^{\mathfrak{B}}}\right), \boldsymbol{P}_{i+1}^{*}\left(\widehat{L}_{-i}, \widehat{\Psi_{i+1}^{\mathfrak{B}}}\right), \ldots, \boldsymbol{P}_{m}^{*}\left(\widehat{L}_{-i}, \widehat{\Psi_{m}^{\mathfrak{B}}}\right)\right]=\arg \max _{\mathfrak{\beta}} \prod_{j \in \mathscr{C}_{i}, j \neq i} v_{j}\left(\widehat{L}_{-i}, \widehat{\Psi_{j}^{\mathfrak{B}}}\right), \\
& \text { s.t. } \quad \sum_{j \in \mathscr{C}_{i}, j \neq i} \boldsymbol{\Re}_{j}^{*}\left(\widehat{L}_{-i}, \widehat{\Psi_{j}^{\mathfrak{B}}}\right) \leq\left(\Delta_{e} \times\left(\eta \times l_{i}\right)\right) .
\end{aligned}
$$


3.3. Fairness-Control Game at the Second Stage. RE is generally defined as energy that comes from resources, such as sunlight, wind, tides, waves, and geothermal heat. To decrease the global greenhouse gas emissions, there are many benefits of using RE sources. For green cellular networks, RE sources can replace conventional energy grid in powering cellular BSs. It is useful not only in the environmental but also in the economic sense while opening opportunities for new business models. Nowadays, it is of great importance to study the RE management in order to determine the potential gains and applicability scenarios $[14,15]$.

Adopting RE in cellular systems affects the planning methodology and architecture of cellular platform. The green cellular network architecture consists of conventional gridpowered BSs, which are also connected by REPs. To maximize the energy efficiency, the joint design and cooperative combination of conventional grid and REPs are critical. In this study, we assume that multiple RE sources exist uniformly, and a BS is connected by only one RE source. Therefore, each BS is connected by dedicated power lines from the conventional power system and one REP. Usually, most of the energy of BS is provided by the conventional grid while receiving subsidized aids from REPs. It is a quite general and real-world applicable architecture [4-6].

Each REP has a set of supporting BSs, called permanent cluster $(\mathscr{M})$. In a $\mathscr{M}$, each BS has its own traffic load while consuming energy differently. Due to the intermittent supply, the main goal of RE management is to fairly distribute the RE in $\mathscr{M}$. Therefore, fairness is a new concern in the RE sharing approach. In computer science, the concept of fairness is related to the amount of delay in servicing a request that can be experienced in a shared resource environment [16]. In green cellular network engineering, fairness is measured whether BSs receive a fair share of $\mathrm{RE}$ provisioning. In general, RE is relatively cheaper compared to electricity from traditional power grid. Therefore, BSs would always prefer using the RE [14]. From the economic viewpoint, each BS should get the same money saving from the RE. From the viewpoint of traffic load balancing, the BS with a heavy traffic load should get the more RE. To characterize the proportional fairness of RE sharing, we follow Jain's fairness index $(\mathscr{F})$; it has been frequently used to measure the fairness of network operations [17]. According to the fundamental idea of $\mathscr{F}$, the economic fairness index $\left(\mathscr{F}_{e_{-} v}\right)$ and the overload fairness index $\left(\mathscr{F}_{o \perp}\right)$ in the $\mathscr{M}^{j}$ are given by

$$
\begin{aligned}
& \mathscr{F}_{e_{-} v}^{j}=\frac{\left(\sum_{i=1}^{w} \gamma_{i}\left(\mathbb{A}^{j}\right)\right)^{2}}{w \times \sum_{i=1}^{w}\left(\gamma_{i}\left(\mathbb{A}^{j}\right)\right)^{2}}, \\
& \mathscr{F}_{o \downarrow}^{j}=\frac{\left(\sum_{i=1}^{w} \mu_{i}\left(\mathbb{A}^{j}\right)\right)^{2}}{w \times \sum_{i=1}^{w}\left(\mu_{i}\left(\mathbb{A}^{j}\right)\right)^{2}},
\end{aligned}
$$

where $w$ and $\mathbb{A}^{j}=\left(\mathscr{A}_{1}^{j}, \mathscr{A}_{2}^{j}, \ldots, \mathscr{A}_{w}^{j}\right)$ are the number of BSs and the RE distribution vector for each BSs in $\mathscr{M}^{j}$, respectively. $\gamma_{i}\left(\mathbb{A}^{j}\right)$ is BS $i$ 's obtained money saving from the $\mathrm{RE}$, and $\mu_{i}\left(\mathbb{A}^{j}\right)$ is the traffic load supported by the traditional power grid. To get the proper combination of $\mathscr{F}_{e_{-} v}^{j}$ and $\mathscr{F}_{o_{-} l}^{j}$, they should be transformed into a single objective function. To provide the best compromise in the presence of different fairness indexes, a multiobjective fairness function $\left(M_{-} \mathscr{F}_{\mathscr{M}}^{j}\right)$ for $\mathscr{M}^{j}$ is developed based on the weighted sum method. By using dynamic joint operations, the developed $M_{-} \mathscr{F}^{j}{ }_{\mathscr{M}}$ is formulated as follows:

$$
M \_\mathscr{F}_{M}^{j}=\left[\beta^{j} \times \mathscr{F}_{e_{-} v}^{j}\right]+\left[\left(1-\beta^{j}\right) \times \mathscr{F}_{o_{-} l}^{j}\right]
$$

where $\beta^{j}$ controls the relative weights given to different fairness indexes. Under diverse network environments, we treat $\beta^{j}$ value decision problem as an online decision problem. When the traffic is uniformly distributed over the BSs in $\mathscr{M}^{j}$, we can put more emphasis on the economic fairness, that is, on $\mathscr{F}_{e_{-} v}^{j}$. In this case, a higher value of $\beta^{j}$ is more suitable. But, if traffic distributions are relatively nonuniform, due to temporal and spatial traffic fluctuations, we should strongly consider the overload fairness, that is, on $\mathscr{F}_{o l}^{j}$. In this case, a lower value of $\beta^{j}$ is more suitable. Therefore, by considering the current traffic profiles of $\mathscr{M}^{j}$, we decide $\beta^{j}$ value as follows:

$$
\beta^{j}=\frac{\min \left\{\mathscr{B}_{l}^{j} \in \mathscr{M}^{j} \mid \phi_{l}^{j}\right\}}{\max \left\{\mathscr{B}_{k}^{j} \in \mathscr{M}^{j} \mid \phi_{k}^{j}\right\}},
$$

where $\mathscr{B}_{k}^{j}$ and $\phi_{k}^{j}$ are the BS $k$ and the current traffic load of BS $k$ in $\mathscr{M}^{j}$. Therefore, in our fair-control algorithm, the value of $\beta$ in each $\mathscr{M}$ is dynamically adjusted to make the system more responsive to current traffic conditions.

3.4. The Main Steps of Proposed Algorithm. In this study, we investigate the BS sleeping and RE distribution algorithms to maximize the performance of green cellular networks. Based on BSs' rationality, these algorithms are formulated as a two-level cooperative game model. Therefore, we assume that BSs would like to join voluntarily the BS sleeping and RE distribution algorithms only when they can get a profit. For the energy efficiency, BSs are grouped dynamically as ad hoc clusters, and a low traffic load BS is switched off to save the energy. Based on the concept of RBS, the saved energy is distributed by active BSs in each cluster. For the energy fairness, we intensively use the RE for BSs. To get a multiobjective fairness, each REP adaptively distributes the RE to its corresponding permanent cluster. The main contribution of our proposed approach is a sophisticated combination of the reciprocal relationship between energy efficiency and fairness; it can provide much more suitable energy sharing scheme. Based on the real-time interactive process, each BS and each REP act strategically to achieve a better profit. In this work, we do not focus on trying to get an optimal solution based on the traditional approach, but instead, an adaptive interactive model is proposed. This approach can dramatically reduce the computational complexity and overheads. Usually, the traditional optimal solutions need exponential time complexity. However, the proposed solution concept only needs polynomial time complexity. The proposed algorithm is described by the following major steps. 
TABLE 1: Application and system parameters used in the simulation experiment.

\begin{tabular}{|c|c|c|c|}
\hline Application type & Maximum service requirement & Minimum service requirement & $\begin{array}{l}\text { Service duration } \\
\text { average/sec }\end{array}$ \\
\hline I & $1.28 \mathrm{Kbps}$ & $0.64 \mathrm{Kbps}$ & $1,200 \mathrm{sec}(20 \mathrm{~min})$ \\
\hline II & $2.56 \mathrm{Kbps}$ & $1.28 \mathrm{Kbps}$ & $1,800 \mathrm{sec}(30 \mathrm{~min})$ \\
\hline III & $3.84 \mathrm{Kbps}$ & $1.92 \mathrm{Kbps}$ & $600 \mathrm{sec}(10 \mathrm{~min})$ \\
\hline IV & $5.12 \mathrm{Kbps}$ & $2.56 \mathrm{Kbps}$ & $900 \mathrm{sec}(15 \mathrm{~min})$ \\
\hline $\mathrm{V}$ & $6.40 \mathrm{Kbps}$ & $3.20 \mathrm{Kbps}$ & $1,800 \mathrm{sec}(30 \mathrm{~min})$ \\
\hline VI & $7.24 \mathrm{Kbps}$ & $3.62 \mathrm{Kbps}$ & $3,000 \mathrm{sec}(50 \mathrm{~min})$ \\
\hline Parameter & Value & \multicolumn{2}{|c|}{ Description } \\
\hline$n$ & 70 & \multicolumn{2}{|c|}{ The number of all BSs in green cellular network } \\
\hline$z$ & 10 & \multicolumn{2}{|c|}{ The number of all REPs in green cellular network } \\
\hline$m, w$ & 7,7 & \multicolumn{2}{|c|}{ The number of BSs in $\mathscr{C}$ and $\mathscr{M}$, respectively } \\
\hline $\mathscr{F}^{\mathfrak{B}}$ & $1 \mathrm{~kW}$ & \multicolumn{2}{|c|}{ The fixed power consumption for each BS } \\
\hline$\eta$ & 1 & \multicolumn{2}{|c|}{ The energy parameters for the traffic load execution } \\
\hline$\varepsilon$ & 0.6 & \multicolumn{2}{|c|}{ The energy parameters for the traffic transferring } \\
\hline$\Delta_{e}$ & 1 & \multicolumn{2}{|c|}{ The cost fee parameters for using electricity } \\
\hline$\Delta_{t}$ & 1.2 & \multicolumn{2}{|c|}{ The cost fee parameters for transferring traffic load } \\
\hline
\end{tabular}

Step 1. At the starting time, all control parameters, that is, $n$, $z, m, w, \mathscr{F}^{\mathfrak{B}}, \eta, \varepsilon, \Delta_{e}$, and $\Delta_{t}$, are chosen from Table 1.

Step 2. Based on the distributed online manner, each BS is monitoring individually its own traffic load and periodically reports this information to the MSC.

Step 3. When a BS's current traffic load is less than $\Gamma_{L}$ or a traffic load in the sleeping BS area is higher than $\Gamma_{H}$, this BS and its neighboring BSs are grouped as a $\mathscr{C}$. In each $\mathscr{C}$, the values of $\Gamma_{L}$ and $\Gamma_{H}$ are dynamically decided according to (2).

Step 4. The MSC makes a BS switching decision, while satisfying the power minimization problem of (1).

Step 5. If the BS is switched off to save energy, the saved energy is dynamically redistributed through RBS in the corresponding $\mathscr{C}$. The preference function $v$ of each neighboring BS in $\mathscr{C}$ is estimated according to (4), and the RBS optimization problem in (5) is solved to get the incentivepayment $\boldsymbol{P}$.

Step 6. In each $\mathscr{M}$ supported by a REP, the RE is adaptively distributed by considering $\mathscr{F}_{e_{-} v}$ and $\mathscr{F}_{o l}$, simultaneously. To get a proper multiobjective fairness, $\beta$ and $M_{-} \mathscr{F}_{\mathscr{M}}$ are obtained using (8) and (7), respectively.

Step 7. To reduce the computation complexity, the amount of energy distribution is specified in terms of basic energy units (BEUs), where one BEU is the minimum amount of the energy readjustment for optimal solution.

Step 8. Individual BS and REP constantly self-monitor the current traffic situation in a distributed online manner; the next iteration resumes at Step 2.

\section{Performance Evaluation}

In this section, we compare the performance of the proposed scheme with other existing schemes $[1,4]$ and confirm the performance superiority of the proposed approach using a simulation model. We have used the simulation tool MATLAB to develop our simulation model. MATLAB is one of the most widely used tools in a number of scientific simulation fields; MATLAB's high-level syntax and dynamic types are ideal for model prototyping. In order to ensure that our simulation model is sufficiently generic to be valid in the real-world, the assumptions used in our simulation are as follows.

(i) Our simulation model was a representation of green cellular network system that included traditional power grid and REPs.

(ii) The simulated system consisted of 70 BSs and 10 REPs for the green cellular platform.

(iii) In each $\mathscr{C}$ or $\mathscr{M}$ cluster, 7 BSs existed, and they were connected simultaneously by the conventional and renewable energy grid.

(iv) In the traffic load service, $1 \mathrm{Kbps}$ service needed $10 \mathrm{~W}$ electrical power.

(v) In wireless cellular networks, traffic services were elastic applications. Application service request was Poisson with rate $\rho$ (services/s) and the range of the offered service load was varied from 0 to 3.0.

(vi) Energy distribution is sequentially negotiated by the size of one BEU, where one BEU is the minimum energy amount (e.g., $10 \mathrm{~W}$ in our system) for the energy readjustment process.

(vii) The RE in each REP was generated randomly from a Gaussian distribution. 
(viii) The service durations of applications were exponentially distributed with different means for different application types.

(ix) Network performance measures obtained based on 100 simulation runs were plotted as a function of the offered traffic load.

(x) The performance criteria obtained through simulation were energy saving ratio, normalized power consumption, and system fairness.

Table 1 shows the system parameters used in the simulation. In order to emulate a real green cellular network system and perform a fair comparison, we used the system parameters for a realistic simulation model.

In this paper, we compare the performance of the proposed scheme with existing schemes: the DCBS scheme [1] and the HEGU scheme [4]. These existing schemes were recently developed as effective green cellular network management algorithms. However, they are successful only in certain circumstances and cannot adaptively estimate the current cellular system conditions. In addition, these schemes operate the cellular network system by some fixed system parameters. Compared to these existing schemes, we can confirm the superiority of our dual-level game approach.

Figure 1 presents the performance comparison of each scheme in terms of energy saving ratio in the green cellular network systems. In this study, the energy saving ratio is used for the performance metric, which is defined as $[1-$ (the ratio between energy consumptions when a number of BSs are turned off and when all BSs are turned on)]. Traditionally, monitoring how the power energy is saved is one of the most critical aspects of green cellular network management. Our proposed scheme adaptively distributes the energy resource to BSs according to the game-based approach. However, the DCBS and HEGU schemes cannot adaptively estimate the current cellular system conditions while causing the extra control overhead. Therefore, we can get a higher energy saving ratio than the other schemes from low to heavy traffic load intensities.

Figure 2 shows the normalized power consumption under different traffic loads. It is clear that the power consumption increases proportional to the number of active BSs; this is because the power consumption mainly comes from the fixed power consumption in each BS. From the simulation results obtained, it is observed that the proposed scheme can adapt to the current traffic condition and effectively reduce the total power consumption. However, other existing schemes can cause potential erroneous decisions under dynamic network environments. In general, lower power consumption is a highly desirable property for real-world green cellular system operations. Under different traffic loads, the proposed scheme can provide a lower power consumption than the other schemes.

The curves in Figure 3 illustrate the system fairness for all the schemes. For fair comparison, we estimate the system fairness of each scheme while considering the economic and overload fairness equally $(\beta=0.5)$. According to the intelligent fairness policy, the proposed scheme

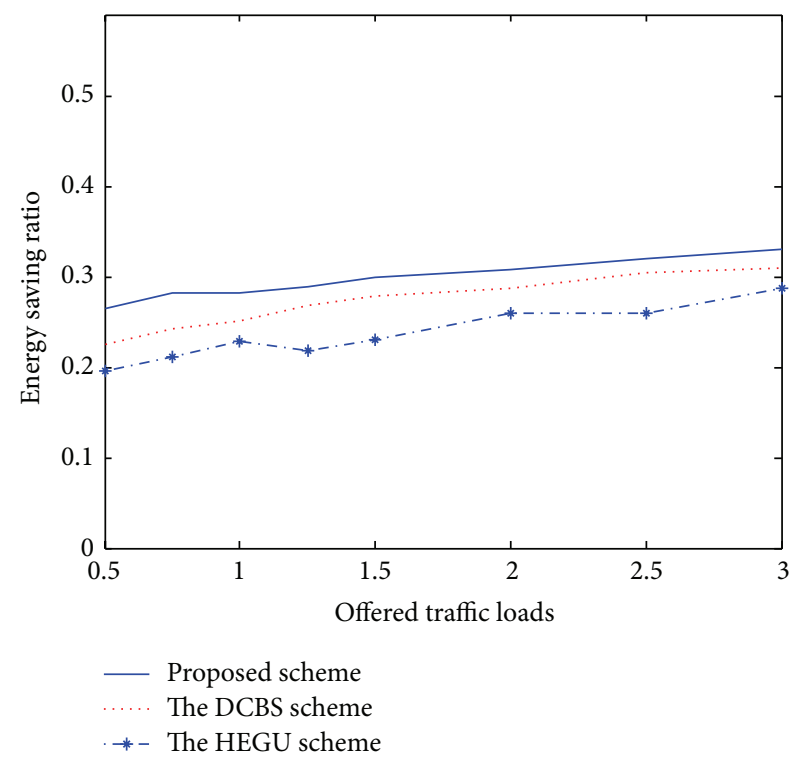

FIGURE 1: Energy saving ratio.

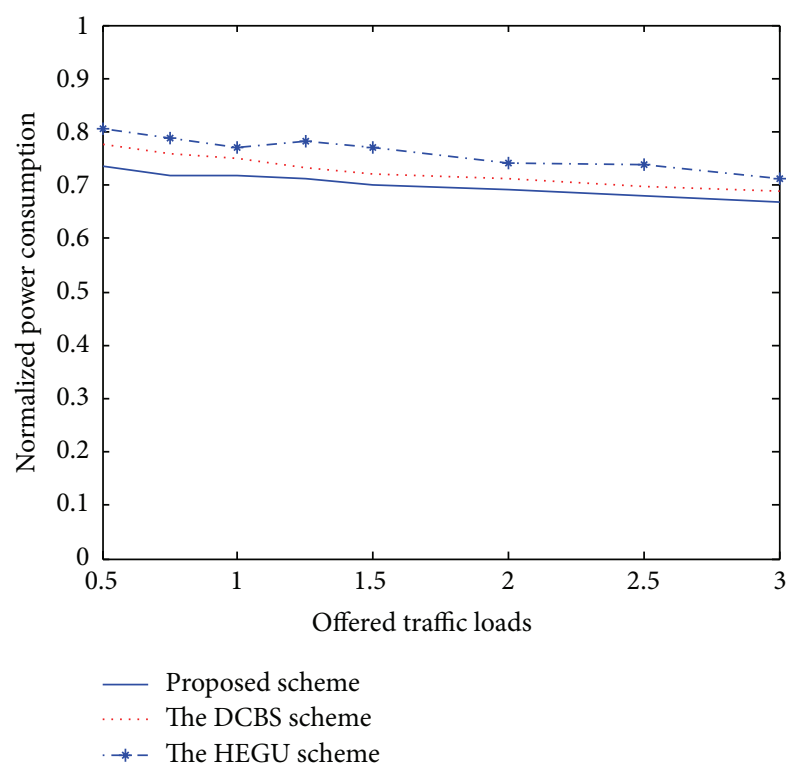

Figure 2: Normalized power consumption.

makes the network system adaptable in order to achieve a balanced energy distribution while ensuring reciprocal fairness. Therefore, the proposed scheme can maintain the excellent system fairness under various traffic load intensities.

The simulation results presented in Figures $1-3$ show that the proposed scheme generally exhibits attractive network performance compared with the other existing schemes $[1,4]$ under widely different traffic load intensities. Due to our adaptive dual-level game approach, we rely on the practical assumptions for real-world cellular system operations. Therefore, the proposed algorithm can get an appropriate performance balance between energy efficiency and fairness, 


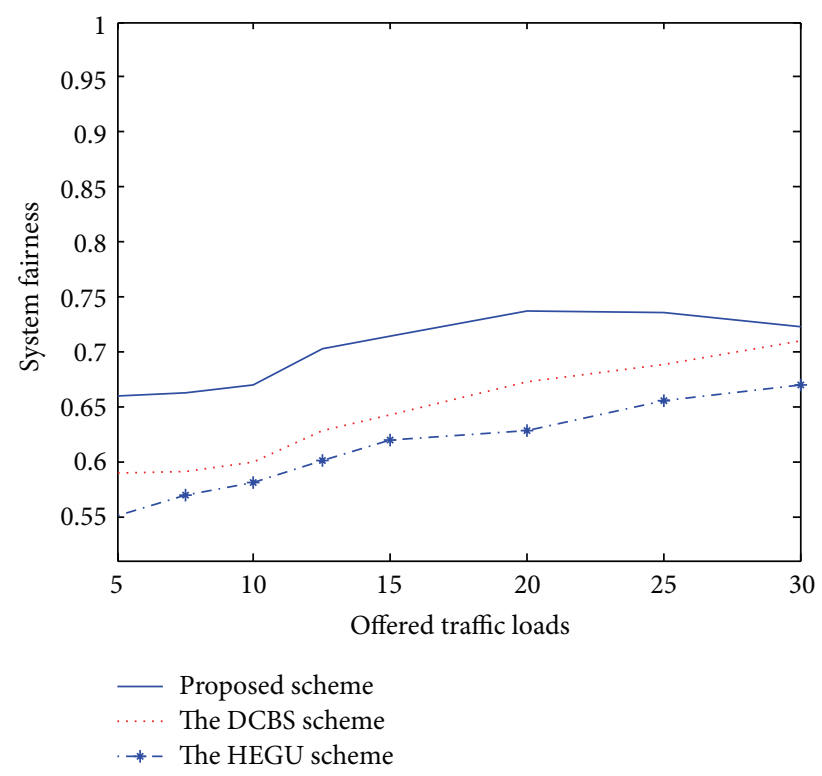

FIGURE 3: System fairness.

while the DCBS scheme [1] and the HEGU scheme [4] cannot offer such an attractive network performance.

\section{Summary and Conclusions}

Research on green cellular networks is quite broad, and a number of research issues and challenges lie ahead. In particular, energy efficiency is a growing concern for cellular system operators to maintain profitability while reducing the overall environment effects. In this study, we have looked into the feasibility of green cellular networks with RE sources. Employing RE not only is environment friendly but also has other benefits with one of the notable points being the shift from energy efficiency to energy fairness. To design a novel energy control scheme, we start from a BS sleeping algorithm to maximize the energy efficiency while ensuring the fairness among BSs. Using two-level game approach, self-regarding BSs are induced to actively participate in the fair-efficient energy control mechanism. By analyzing the simulation results, it can be concluded that the proposed scheme can effectively deal with the energy distribution problem in green cellular networks compared to other existing schemes. For future research, we need to investigate the design of energy aware heterogeneous networks, where the high-power macrocell BSs and low-power femtocell BSs coexist. In addition, to further reduce the energy consumption, we are also looking on the optimal cell size decisions and femtocell BS locations taking into consideration the energy spent for the system backhaul and signaling overhead.

\section{Competing Interests}

The author declares that there are no competing interests regarding the publication of this paper.

\section{Authors' Contributions}

Sungwook Kim is a sole author of this work and ES (i.e., participated in the design of mathematical equations, the implementation of proposed algorithm, and compared the performance of the proposed scheme with other existing schemes).

\section{Acknowledgments}

This research was supported by the MSIP (Ministry of Science, ICT and Future Planning), Korea, under the ITRC (Information Technology Research Center) support program (IITP-2016-H8501-16-1018) supervised by the IITP (Institute for Information \& Communications Technology Promotion) and was supported by Basic Science Research Program through the National Research Foundation of Korea (NRF) funded by the Ministry of Education (NRF2015R1D1A1A01060835).

\section{References}

[1] J. Zheng, Y. Cai, X. Chen, R. Li, and H. Zhang, "Optimal base station sleeping in green cellular networks: a distributed cooperative framework based on game theory," IEEE Transactions on Wireless Communications, vol. 14, no. 8, pp. 4391-4406, 2015.

[2] J. Wu, Y. Zhang, M. Zukerman, and E. K.-N. Yung, "Energyefficient base-stations sleep-mode techniques in green cellular networks: a survey," IEEE Communications Surveys \& Tutorials, vol. 17, no. 2, pp. 803-826, 2015.

[3] B.-H. Park, Y. Kim, B.-D. Kim, T. Hong, S. Kim, and J. K. Lee, "High performance computing: infrastructure, application, and operation," Journal of Computing Science and Engineering, vol. 6, no. 4, pp. 280-286, 2012.

[4] T. Han and N. Ansari, "On optimizing green energy utilization for cellular networks with hybrid energy supplies," IEEE Transactions on Wireless Communications, vol. 12, no. 8, pp. $3872-$ 3882, 2013.

[5] Y.-K. Chia, S. Sun, and R. Zhang, "Energy cooperation in cellular networks with renewable powered base stations," IEEE Transactions on Wireless Communications, vol. 13, no. 12, pp. 6996-7010, 2014.

[6] N. Reyhanian, V. Shah-Mansouri, B. Maham, and C. Yuen, "Renewable energy distribution in cooperative cellular networks with energy harvesting," in Proceedings of the 26th Annual International Symposium on Personal, Indoor, and Mobile Radio Communications (PIMRC '15), pp. 1617-1621, Hong Kong, September 2015.

[7] J. Xiao and R. Boutaba, "The design and implementation of an energy-smart home in Korea," Journal of Computing Science and Engineering, vol. 7, no. 3, pp. 204-210, 2013.

[8] S. Kim, Game Theory Applications in Network Design, IGI Global, Pennsylvania, Pa, USA, 2014.

[9] T. Mao, G. Feng, L. Liang, S. Qin, and B. Wu, "Distributed energy-efficient power control for macro-femto networks," IEEE Transactions on Vehicular Technology, vol. 65, no. 2, pp. 718-731, 2016.

[10] L. B. Le, "QoS-aware BS switching and cell zooming design for OFDMA green cellular networks," in Proceedings of the IEEE Global Communications Conference (GLOBECOM '12), pp. 1544-1549, IEEE, Anaheim, Calif, USA, December 2012. 
[11] D. Li, W. Saad, I. Guvenc, A. Mehbodniya, and F. Adachi, "Decentralized energy allocation for wireless networks with renewable energy powered base stations," IEEE Transactions on Communications, vol. 63, no. 6, pp. 2126-2142, 2015.

[12] Y. Bao, J. Wu, S. Zhou, and Z. Niu, "Bayesian mechanism based inter-operator base station sharing for energy saving," in Proceedings of the IEEE International Conference on Communications (ICC '15), pp. 49-54, London, UK, 2015.

[13] H. Zhang, J. Cai, and X. Li, "Energy-efficient base station control with dynamic clustering in cellular network," in Proceedings of the 8th IEEE International ICST Conference on Communications and Networking in China (CHINACOM '13), pp. 384-388, Guilin, China, August 2013.

[14] C. Zhang, W. Wu, H. Huang, and H. Yu, "Fair energy resource allocation by minority game algorithm for smart buildings," in Proceedings of the 15th Design, Automation and Test in Europe Conference and Exhibition (DATE '12), pp. 63-68, March 2012.

[15] H. Al Haj Hassan, L. Nuaymi, and A. Pelov, "Renewable energy in cellular networks: a survey," in Proceedings of the IEEE Online Conference on Green Communications (GreenCom '13), pp. 1-7, IEEE, Piscataway, NJ, USA, October 2013.

[16] M. Etinski and A. Schulke, "Fair sharing of RES among multiple users," in Proceedings of the IEEE Power and Energy Society Innovative Smart Grid Technologies Conference (ISGT '14), pp. 1-5, Washington, DC, USA, February 2014.

[17] M. Dianati, X. Shen, and S. Naik, "A new fairness index for radio resource allocation in wireless networks," in Proceedings of the IEEE Wireless Communications and Networking Conference (WCNC '05), vol. 2, pp. 712-715, March 2005. 

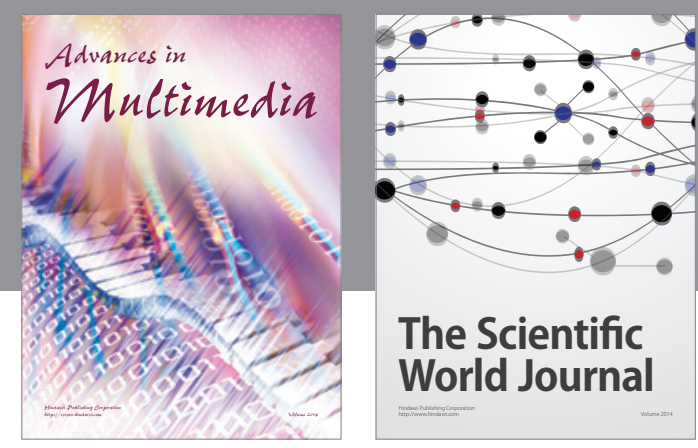

The Scientific World Journal
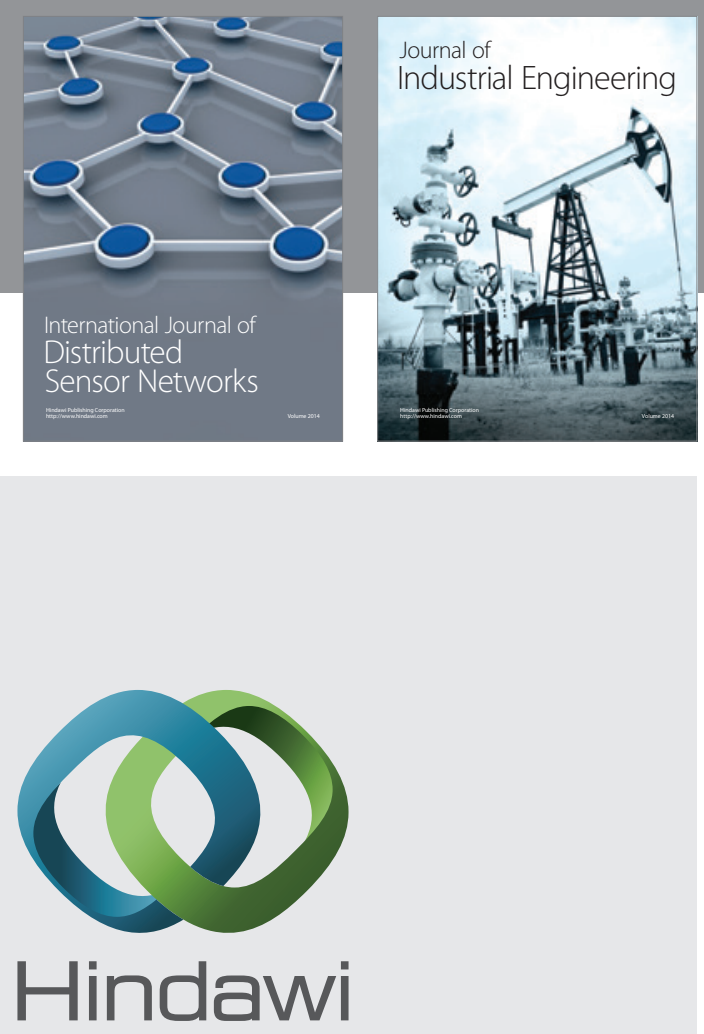

Submit your manuscripts at

http://www.hindawi.com

\section{Computer Networks} and Communications
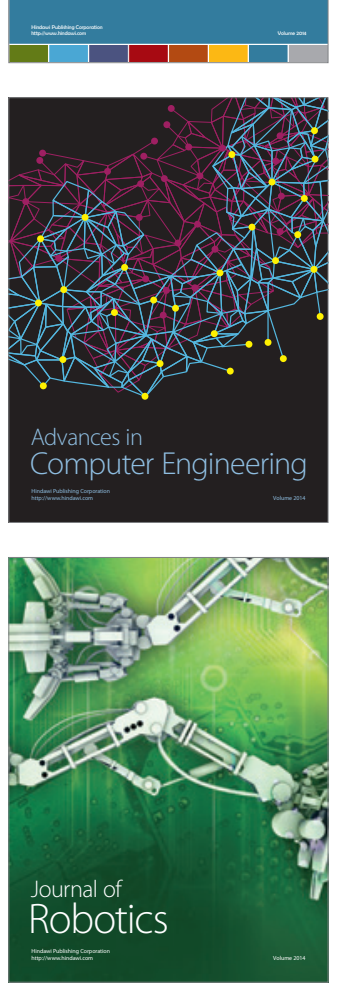
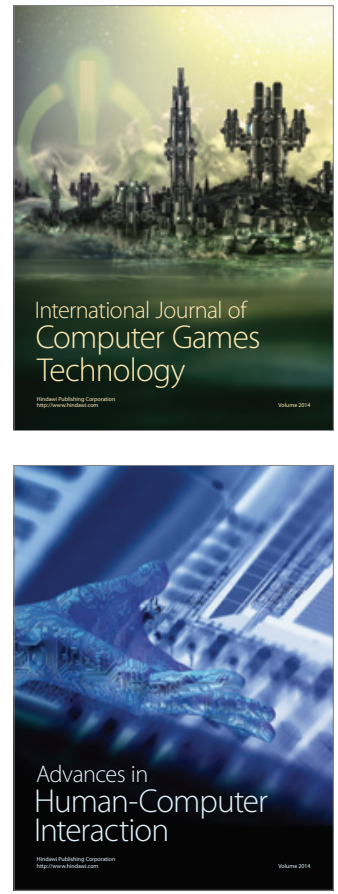
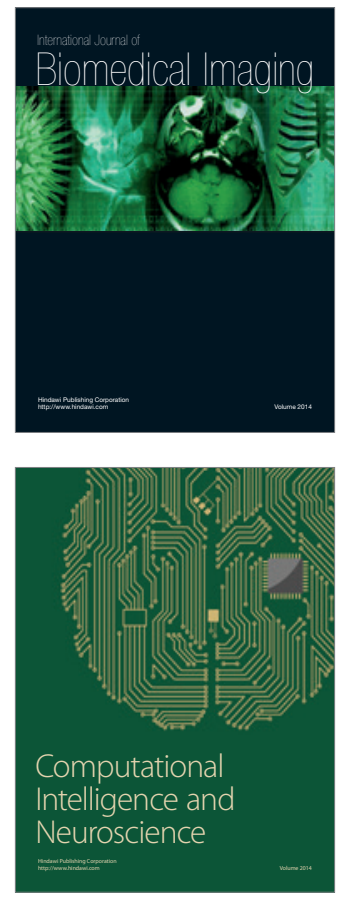
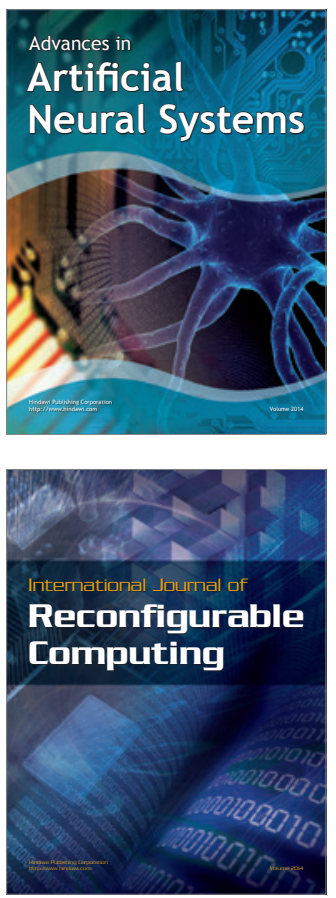
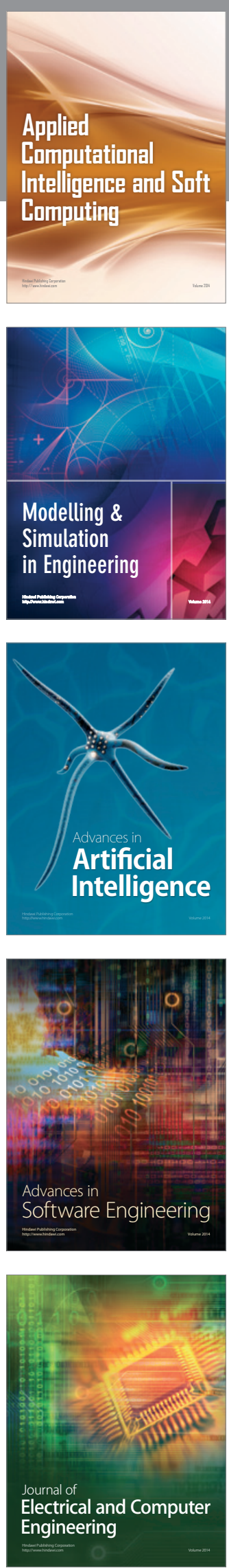